\title{
Ultrasound evaluation of diaphragm function in mechanically ventilated patients: comparison to phrenic stimulation and prognostic implications
}

\author{
Bruno-Pierre Dubé, ${ }^{1,2}$ Martin Dres, ${ }^{1,3}$ Julien Mayaux, ${ }^{3}$ Suela Demiri, ${ }^{3}$ \\ Thomas Similowski, ${ }^{1,3}$ Alexandre Demoule ${ }^{1,3}$
}

- Additional material is published online only. To view please visit the journal online (http://dx.doi.org/10.1136/ thoraxjnl-2016-209459).

${ }^{1}$ Sorbonne Universités, UPMC Univ Paris 06, INSERM, UMRS1158 Neurophysiologie respiratoire expérimentale et clinique, Paris, France

${ }^{2}$ Département de médecine, service de pneumologie, Hôpital Hôtel-Dieu du Centre Hospitalier de l'Université de Montréal (CHUM), Montréal, Québec, Canada

${ }^{3}$ AP-HP, Groupe Hospitalier Pitié-Salpêtrière Charles Foix, Service de Pneumologie et Réanimation Médicale (Département "R3S"), F-75013, Paris, France

\section{Correspondence to}

Dr Alexandre Demoule, Service de Pneumologie et Réanimation Médicale, Groupe Hospitalier Pitié-Salpêtrière, 47-83 boulevard de I'Hôpital, 75651 Paris Cedex 13, France; alexandre.demoule@aphp.fr

B-PD and MD contributed equally

Received 20 September 2016 Revised 17 February 2017 Accepted 22 February 2017 Published Online First 30 March 2017

\section{ABSTRACT}

Rationale In intensive care unit (ICU) patients, diaphragm dysfunction is associated with adverse clinical outcomes. Ultrasound measurements of diaphragm thickness, excursion (EXdi) and thickening fraction (TFdi) are putative estimators of diaphragm function, but have never been compared with phrenic nerve stimulation. Our aim was to describe the relationship between these variables and diaphragm function evaluated using the change in endotracheal pressure after phrenic nerve stimulation (Ptr,stim), and to compare their prognostic value.

Methods Between November 2014 and June 2015, Ptr, stim and ultrasound variables were measured in mechanically ventilated patients $<24$ hours after intubation ('initiation of mechanical ventilation (MV)', under assist-control ventilation, $\mathrm{ACV}$ ) and at the time of switch to pressure support ventilation ('switch to PSV'), and compared using Spearman's correlation and receiver operating characteristic curve analysis. Diaphragm dysfunction was defined as Ptr, stim $<11 \mathrm{~cm} \mathrm{H}_{2} \mathrm{O}$. Results 112 patients were included. At initiation of MV, Ptr, stim was not correlated to diaphragm thickness $(p=0.28)$, EXdi $(p=0.66)$ or TFdi $(p=0.80)$. At switch to PSV, TFdi and EXdi were respectively very strongly and moderately correlated to Ptr,stim, $(r=0.87, p<0.001$ and $0.45, p=0.001)$, but diaphragm thickness was not $(p=0.45)$. A TFdi $<29 \%$ could reliably identify diaphragm dysfunction (sensitivity and specificity of $85 \%$ and $88 \%$ ), but diaphragm thickness and EXdi could not. This value was associated with increased duration of ICU stay and MV, and mortality.

Conclusions Under ACV, diaphragm thickness, EXdi and TFdi were uncorrelated to Ptr,stim. Under PSV, TFdi was strongly correlated to diaphragm strength and both were predictors of remaining length of MV and ICU and hospital death.

\section{INTRODUCTION}

Diaphragm dysfunction has become a leading concern in mechanically ventilated patients because of its high prevalence ${ }^{1-4}$ and its association with negative clinical outcomes. In particular, its presence in this population has been associated with the increased duration of mechanical ventilation (MV), ${ }^{5}{ }^{6}$ weaning failure $^{378}$ and death. ${ }^{19}$ Accordingly, a reliable and simple diagnostic method to quantify diaphragm function in the intensive care unit (ICU) setting would be a desirable tool for clinicians in order to
Key messages

What is the key question?

- In critically ill patients undergoing mechanical ventilation (MV), are ultrasound diaphragm measurements reliable estimators of diaphragm function as assessed with the gold standard phrenic stimulation technique (Ptr,stim) and are they associated with clinical outcomes?

What is the bottom line?

- Neither diaphragm thickness, its excursion or its thickening fraction (TFdi) were correlated to diaphragm function at the moment of initiation of MV, but TFdi alone was strongly correlated to Ptr, stim and to clinical outcome at the moment of switch to pressure support ventilation.

\section{Why read on?}

- This study is the first to systematically compare various ultrasonographic markers of diaphragm function to phrenic nerve stimulation in MV patients, providing a better understanding of the conditions in which these markers can act as reliable estimators of diaphragm function and clinical outcome in this population.

guide the management of MV, evaluate prognosis and possibly improve the outcome of patients with diaphragm dysfunction. ${ }^{10-12}$

The reference method to evaluate diaphragm function remains the measurement of the negative intrathoracic pressure generated by bilateral stimulation of the phrenic nerves. ${ }^{13}$ However, this technique requires specialised expertise and is time-consuming, and is therefore impractical for routine use in the clinical setting. Recently, ultrasound has emerged as a promising technique allowing easy and rapid estimation of diaphragm function, especially in the weaning period. ${ }^{14-18}$ Diaphragm thickness has been shown to decline in response to prolonged $\mathrm{MV}$, a finding that presumably reflects diaphragmatic fibre atrophy. ${ }^{19}$ The inspiratory thickening of the diaphragm has been more extensively correlated with relevant outcomes such as weaning failure and has been proposed as a dynamic marker of diaphragm function. ${ }^{20}$ 
However, these indices have not been directly compared with an established marker of diaphragm function.

In light of these findings, our objectives were to (1) study the relationship between ultrasound-derived markers of diaphragm function and negative intrathoracic pressure generated by stimulation of the phrenic nerves (diaphragm twitch) in critically ill patients receiving MV, (2) establish which ultrasound-derived marker is more reliable at quantifying diaphragm function and (3) evaluate whether ultrasound-derived markers of diaphragm function are associated with clinical prognosis.

Some of the data from this study have already been published as an abstract. $^{21}$

\section{PATIENTS AND METHODS}

The study was conducted from November 2014 to June 2015 in a 10-bed ICU within an 1800-bed university hospital. The protocol was approved by the Comité de Protection des Personnes Ile de France VI. Informed consent was obtained from patients or their relatives. Thirty-five patients from this study were also enrolled in a previous study by our group. ${ }^{22}$

\section{Patients}

Patients were eligible for inclusion as soon as intubation was completed and if they had an expected duration of MV of $>24$ hours. Exclusion criteria were: contraindications to magnetic stimulation of the phrenic nerves (cardiac pacemaker/defibrillator, cervical implants), suspicion of underlying hemidiaphragm paresis (defined as an elevation of $>2.5 \mathrm{~cm}$ of one hemidiaphragm compared with the other on chest radiograph), pre-existing neuromuscular disorders, cervical spine injury, pregnancy, age $<18$ years and decision to withhold lifesustaining treatment.

\section{Protocol}

Diaphragm function was assessed with two techniques: (1) measurement of pressure generating capacity in response to bilateral magnetic stimulation of the phrenic nerves and (2) ultrasound measurement of diaphragm thickness, excursion (EXdi) and thickening fraction (TFdi). Whenever possible, diaphragm assessment was performed for each patient at two time points: (1) within 24 hours of intubation, while patients were receiving assist-control ventilation (ACV) ('initiation of mechanical ventilation') and (2) as soon as patients could sustain pressure support ventilation (PSV, termed 'switch to PSV') for at least 1 hour (see online supplemental material, for description of the criteria used to assess tolerance to PSV). When initiated, pressure support was titrated to target a tidal volume of 6$8 \mathrm{~mL} / \mathrm{kg}$ ideal body weight.

\section{Diaphragm assessment}

Diaphragm assessment was performed at the aforementioned time points unless a transient condition compromising the reliability of the measurements was present. These conditions were (1) the use of neuromuscular blocking agents within the preceding 24 hours (except for succinylcholine used during rapidsequence intubation) and (2) factors interfering with phrenic nerve stimulation (multiple functioning chest drains, high intrinsic positive end-expiratory pressure (PEEP), see below).

\section{Phrenic nerves stimulation}

The pressure generating capacity of the diaphragm was assessed in terms of the change in endotracheal tube pressure induced by bilateral anterior magnetic phrenic nerve stimulation (Ptr,stim), as already described ${ }^{4} 2324$ (see online supplemental material).
Stimulations were delivered at the maximum intensity allowed by the stimulator, which has been showed to result in supramaximal stimulation in the majority of cases. ${ }^{1492425}$

\section{Ultrasound assessment of diaphragmatic thickness, EXdi and thickening}

Ultrasound measurements were performed by one of the two first authors. Measurements were initially attempted on both hemidiaphragms, but evaluation of the left side was abandoned after the first 25 patients because of lower interobserver agreement (see online supplemental material, e-table 1). The measurement of EXdi was added to the protocol 3 months after the beginning of the study, by which time 51 patients had already been recruited. The interobserver reliability of the ultrasound measurements of the right hemidiaphragm between the two authors has already been reported, with intraclass correlation coefficients for the measurement of end-expiratory and end-inspiratory diaphragm thickness and diaphragm TFdi all $>0.87 .^{22}$

Assessment of diaphragm thickness, EXdi and thickening was performed using a $4-12 \mathrm{MHz}$ linear array transducer (Sparq ultrasound system, Phillips, Philips Healthcare, Andover, Massachusetts, USA) while patients remained connected to the ventilator. As previously reported, ${ }^{26}$ the probe was placed perpendicular to the right chest wall, at the midaxillary line between the 9th and 10th right intercostal spaces (at the level of the zone of apposition) and the diaphragm was identified as a three-layered structure comprising two hyperechoic lines representing the pleural and peritoneal membranes and a middle hypoechoic layer representing the diaphragmatic muscle. Using M-mode at a sweep speed of $10 \mathrm{~mm} / \mathrm{s}$, at least three breathing cycles were recorded. Diaphragm thickness was measured at end-expiration and end-inspiration using electronic callipers. TFdi was calculated as ((end-inspiratory thickness end-expiratory thickness)/end-expiratory thickness). Three uninterrupted and undisturbed breathing cycles were evaluated, and the average of the individual values was reported. Diaphragm EXdi was measured using M-mode, by placing the probe in the right subcostal region and targeting the beam at the highest point of the diaphragmatic dome. ${ }^{26}$

\section{Clinical data collection}

Demographic and physiological variables and medication were recovered from the medical charts of the patients. Sepsis was identified according to current guidelines. ${ }^{27}$ The duration of MV, time to successful extubation, ICU and hospital stay, and ICU and hospital mortality were recorded. We defined successful extubation as extubation not followed by re-intubation within 48 hours.

\section{Outcomes}

Total duration of MV and ICU stay, remaining duration of MV after measurement at switch to PSV and ICU and hospital death were used as clinical outcomes. Predictor variables are listed in the Statistical analysis section.

\section{Statistical analysis}

Normality of the data was evaluated with the Kolmogorov-Smirnov test. Continuous variables are presented as median and IQR and categorical variables are expressed as absolute and relative frequencies. Mixed linear regression analyses were used to compare variables measured at initiation of MV to those measured at switch to PSV. t-tests, Mann-Whitney $\mathrm{U}$ tests or $\chi^{2}$ tests, where appropriate, were used to compare 


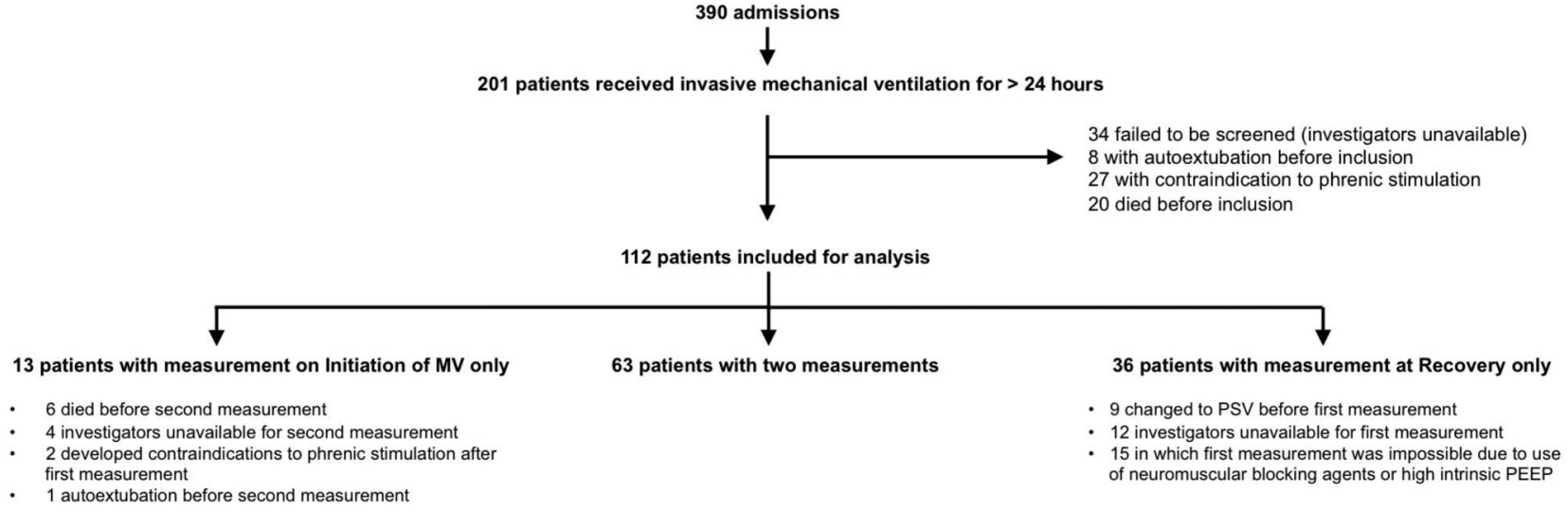

Figure 1 Study flow chart. MV, mechanical ventilation; PEEP, positive end-expiratory pressure; PSV, pressure support ventilation.

outcomes between subgroups of patients. Relationships between diaphragm thickness, EXdi, TFdi and Ptr,stim were assessed using linear regression analysis and Spearman's correlation. Receiver operating characteristic (ROC) curve analyses were performed to identify optimal cut-off values of diaphragm thickness, EXdi and TFdi in predicting diaphragm dysfunction, and these estimates were cross-validated using bootstrapping with 1000 replications. Diaphragm dysfunction was defined as Ptr, stim $<11 \mathrm{~cm} \mathrm{H}_{2} \mathrm{O}^{1-4} 13$

To assess the prognostic value of diaphragm measurements, univariate analyses were performed to evaluate the relationship between clinically relevant variables and selected outcomes. Predictor variables included age, gender, COPD, diabetes, cirrhosis, tobacco smoking, Sepsis-related Organ Failure Assessment Score on admission, PEEP level, tidal volume, respiratory rate, arterial pO2 $\left(\mathrm{PaO}_{2}\right) /$ fraction of inspired oxygen $\left(\mathrm{FiO}_{2}\right)$ ratio, arterial pCO2, Ptr,stim, diaphragm thickness and TFdi. Then, for each outcome, two separate multiple linear regressions or binary logistic regressions were performed. Ptr, stim and TFdi were each entered into separate multivariate analyses, along with other variables with a $\mathrm{p}$ value of $<0.05$ in univariate analysis, to identify independent predictors of clinical outcomes.

Statistical significance was defined as $\mathrm{p} \leq 0.05$. Analyses were performed using SPSS V.21 (SPSS, Chicago, Illinois, USA).

\section{RESULTS}

\section{Population}

During the study period, 390 patients were admitted to the ICU (figure 1). Among them, 112 patients met inclusion criteria and were included in the study. Characteristics of patients on inclusion are displayed in table 1 .

Seventy-six patients were studied at initiation of MV (under $\mathrm{ACV}$, of which 68 did not spontaneously trigger the ventilator) and 99 at time to switch to PSV. Characteristics of the patients at these two time points are presented in table 2 .

Additional description of the patients according to the number of measurements performed is displayed in the online supplemental material (e-table 2). At switch to PSV, patients were receiving a median pressure support level of 10 (8-11) $\mathrm{cm} \mathrm{H}_{2} \mathrm{O}$, and TFdi was significantly correlated to tidal volume, even after controlling for the level of pressure support (partial correlation coefficient $0.54, \mathrm{p}<0.001$ ). The prevalence of diaphragm dysfunction was $77 \%$ at initiation of MV and $68 \%$ at switch to PSV. EXdi was measured in 22 patients at initiation of MV and 50 patients at switch to PSV.
Table 1 Characteristics of the study population

\begin{tabular}{ll}
\hline & $\mathrm{n}=112$ \\
\hline Gender, men & $76(68)$ \\
Age, years & $62(49-71)$ \\
BMI, $\mathrm{kg} / \mathrm{m}^{2}$ & $24(22-29)$ \\
Tobacco smoking, $\mathrm{n}(\%)$ & $48(43)$ \\
Comorbidities & \\
$\quad$ COPD, $\mathrm{n}(\%)$ & $24(21)$ \\
Diabetes, $\mathrm{n}(\%)$ & $24(21)$ \\
Cirrhosis, $\mathrm{n}(\%)$ & $17(15)$ \\
Sepsis on admission to ICU, $\mathrm{n}(\%)$ & $51(46)$ \\
SAPS 2 & $55(33-71)$ \\
Primary indication for mechanical ventilation & \\
Shock, $\mathrm{n}(\%)$ & $52(46)$ \\
Coma, $\mathrm{n}(\%)$ & $25(22)$ \\
Acute respiratory failure, $\mathrm{n}$ (\%) & $37(32)$ \\
\hline Continuous variables are presented as median (IQR) and categorical variables as \\
absolute and relative frequency. \\
BMI, body mass index; ICU, intensive care unit; SAPS 2, Simplified Acute Physiology \\
Score.
\end{tabular}

\section{Relationship between Ptr,stim and ultrasound measurements}

At initiation of MV, there was no relationship between Ptr,stim and diaphragm thickness $(\beta=-0.17, p=0.16$, Spearman's $r=$ $-0.13,95 \% \mathrm{CI}-0.35$ to $0.10, p=0.28)$, or TFdi $(\beta=-0.09$, $\mathrm{p}=0.46$, Spearman's $\mathrm{r}=-0.09,95 \% \mathrm{CI}-0.31$ to $0.14, \mathrm{p}=0.45$ ) (figure 2). There was also no relationship between Ptr,stim and EXdi $(\beta=0.05, p=0.81$, Spearman's $r=0.10,95 \% \mathrm{CI}-0.13$ to $0.32, \mathrm{p}=0.66$ ) (e-figure 1).

At switch to PSV, there was no relationship between Ptr,stim and diaphragm thickness $(\beta=-0.01, p=0.92$, Spearman's $r=-0.03$, $95 \% \mathrm{CI}-0.23$ to $0.17, \mathrm{p}=0.80$ ) (figure 3 ). However, there were statistically significant associations and very strong and moderate correlations, respectively, between Ptr,sim and TFdi $(\beta=0.89$, $\mathrm{p}<0.001$, Spearman's $\mathrm{r}=0.87,95 \%$ CI 0.81 to $0.91, \mathrm{p}<0.001)$ (figure 3) and EXdi ( $\beta=0.28, p=0.05$, Spearman's $r=0.45,95 \%$ CI 0.20 to $0.65, \mathrm{p}=0.001$ ) (e-figure 1 ).

\section{Diagnosis of diaphragm dysfunction using ultrasound markers}

At initiation of MV, neither diaphragm thickness, EXdi nor TFdi could reliably identify the presence of diaphragm 
Table 2 Characteristics of the patients according to number and time of measurements

\begin{tabular}{|c|c|c|c|}
\hline & Initiation of MV ( $n=76)$ & Switch to PSV (n=99) & p Value \\
\hline Days from intubation, days & $1(1-1)$ & $4(2-6)$ & $<0.001$ \\
\hline \multicolumn{4}{|l|}{ Ventilator mode } \\
\hline Assist-control ventilation & $76(100)$ & $0(0)$ & - \\
\hline Pressure support ventilation & $0(0)$ & $99(100)$ & - \\
\hline \multicolumn{4}{|l|}{ Ventilatory variables } \\
\hline Spontaneous triggering of the ventilator, ${ }^{*} \mathrm{n}(\%)$ & $8(11)$ & $99(100)$ & $<0.001$ \\
\hline Pressure support level, $\mathrm{cm} \mathrm{H}_{2} \mathrm{O}$ & NA & $10(8-11)$ & - \\
\hline PEEP level, $\mathrm{cm} \mathrm{H}_{2} \mathrm{O}$ & $5(5-8)$ & $5(5-7)$ & 0.26 \\
\hline Tidal volume, $\mathrm{mL} / \mathrm{kg}$ ideal body weight & $6.4(6.1-7.0)$ & $7.1(6.0-8.4)$ & $<0.001$ \\
\hline Respiratory rate, breaths/min & $21(18-23)$ & $21(19-23)$ & 0.99 \\
\hline \multicolumn{4}{|l|}{ Arterial blood gases } \\
\hline $\mathrm{pH}$ & $7.36(7.28-7.44)$ & $7.44(7.37-7.46)$ & $<0.001$ \\
\hline $\mathrm{PaO}_{2} / \mathrm{FiO}_{2}$ ratio, $\mathrm{mm} \mathrm{Hg}$ & $222(163-280)$ & $260(209-333)$ & 0.02 \\
\hline $\mathrm{PaCO}_{2}, \mathrm{~mm} \mathrm{Hg}$ & $40(33-47)$ & $38(34-47)$ & 0.91 \\
\hline Blood lactate, $\mathrm{mmol} / \mathrm{L}$ & $1.8(1.1-2.8)$ & $1.5(1.1-1.9)$ & 0.01 \\
\hline Bicarbonates, $\mathrm{mmol} / \mathrm{L}$ & $22(18-25)$ & $25(22-28)$ & $<0.001$ \\
\hline Mean arterial pressure, $\mathrm{mm} \mathrm{Hg}$ & $78(70-89)$ & $81(71-96)$ & 0.25 \\
\hline Heart rate, bpm & $87(71-103)$ & $90(78-104)$ & 0.17 \\
\hline \multicolumn{4}{|l|}{ Active medication } \\
\hline Benzodiazepines, n (\%) & $33(43)$ & $34(34)$ & 0.12 \\
\hline Propofol, n (\%) & $38(50)$ & $47(48)$ & 0.58 \\
\hline Opiates, n (\%) & $53(70)$ & $47(48)$ & 0.004 \\
\hline Norepinephrine, n (\%) & $46(61)$ & $36(36)$ & 0.001 \\
\hline Corticosteroids, n (\%) & $13(17)$ & $13(13)$ & 0.20 \\
\hline
\end{tabular}

Continuous variables are presented as median (IQR) and categorical variables are expressed as absolute and relative frequency.

*In assist-control ventilation, spontaneous triggering of the ventilator was considered present when the observed respiratory rate was higher than the respiratory rate set on the ventilator.

$\mathrm{FiO}_{2}$, fraction of inspired oxygen; MV, mechanical ventilation; $\mathrm{NA}$, not applicable; $\mathrm{PaCO}_{2}$, arterial $\mathrm{pCO} 2 ; \mathrm{PaO}_{2}$, arterial pO2; $\mathrm{PEEP}$, positive end-expiratory pressure; PSV, pressure support ventilation.
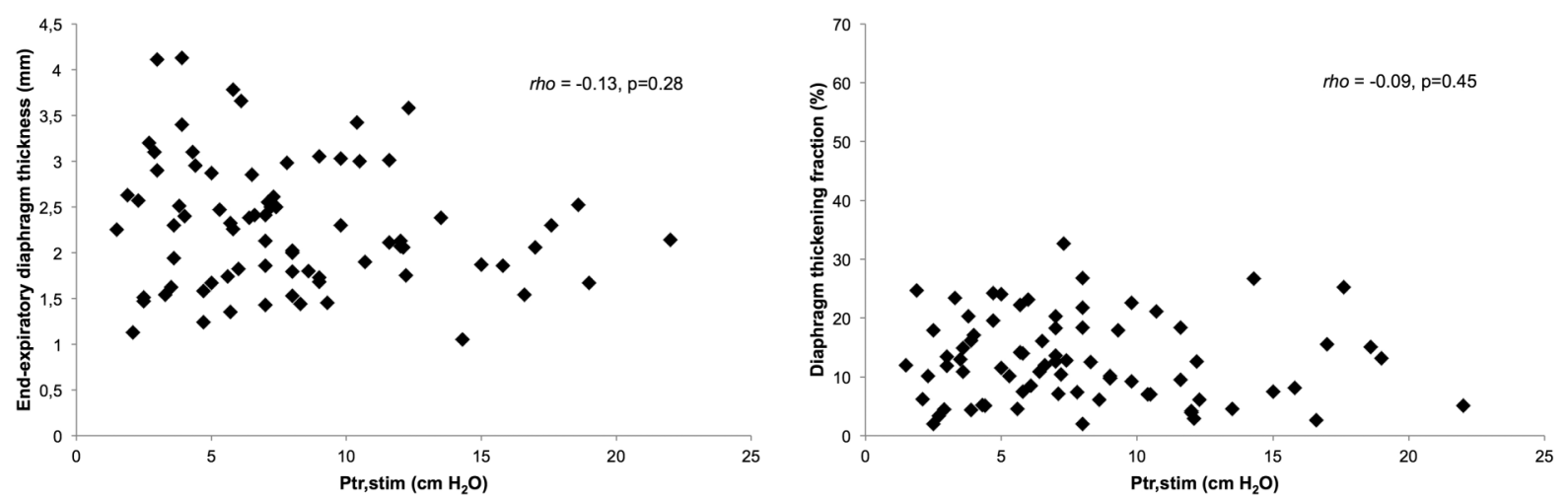

Figure 2 Correlation analysis between changes in endotracheal tube pressure induced by bilateral anterior magnetic phrenic nerve stimulation during manual airway occlusion (Ptr,stim) and end-expiratory diaphragm thickness (left panel) and thickening fraction (right panel) measured by ultrasound on initiation of mechanical ventilation.

dysfunction (area under ROC curve $0.58,95 \%$ CI 0.44 to 0.72 , $\mathrm{p}=0.33,0.60,95 \%$ CI 0.35 to $0.86, \mathrm{p}=0.43$ and $0.63,95 \% \mathrm{CI}$ 0.46 to $0.79, \mathrm{p}=0.12$, respectively) (figure 4 and e-figure 2 ). At switch to PSV, TFdi could reliably identify diaphragm dysfunction (area under ROC curve 0.91 , 95\% CI 0.85 to 0.97 , $\mathrm{p}<0.001$ ), whereas diaphragm thickness and EXdi could not (area under ROC curve $0.55,95 \%$ CI 0.43 to $0.67, \mathrm{p}=0.44$ and $0.63,95 \%$ CI 0.48 to $0.78, p=0.12$, respectively) (figure 4 and e-figure 2). At switch to PSV, a TFdi <29\% (bootstrapped $95 \%$ CI $25 \%$ to $30 \%$ ) could detect diaphragm dysfunction with a sensitivity of $85 \%$ and specificity of $88 \%$, with positive and negative values of $93 \%$ and $74 \%$, respectively.

\section{Comparison of the prognostic values of Ptr,stim and TFdi}

The prognostic values of Ptr,stim and TFdi were measured and compared at switch to PSV since it was the only time point at which an ultrasound measurement could reliably predict diaphragm dysfunction. The threshold value of $29 \%$ for the identification of diaphragm dysfunction had a similar prognostic value as Ptr,stim $<11 \mathrm{~cm} \mathrm{H}_{2} \mathrm{O}$, with both being associated with worse outcome regarding length of $\mathrm{MV}$ and ICU stay, remaining length of MV after the measurement, and ICU and hospital mortality (table 3).

e-table 3 shows the factors associated with remaining duration of MV identified by univariate analysis. By multiple linear regression model analysis, two of these factors independently 

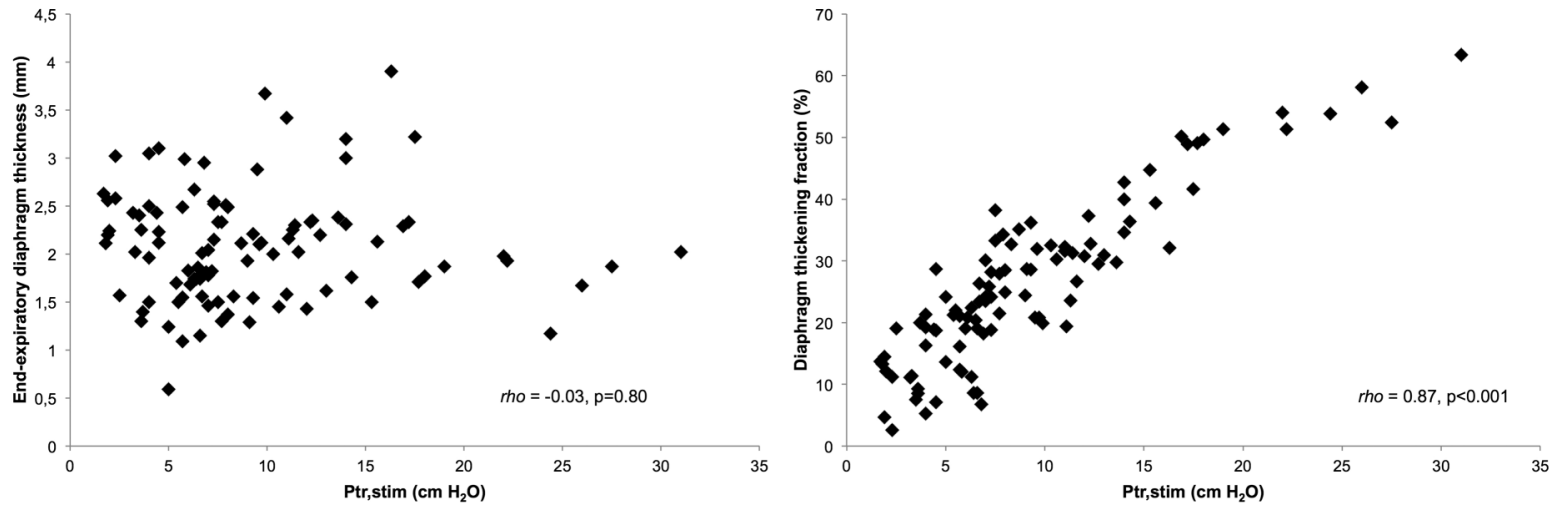

Figure 3 Correlation analysis between changes in endotracheal tube pressure induced by bilateral anterior magnetic phrenic nerve stimulation during manual airway occlusion (Ptr,stim) and end-expiratory diaphragm thickness (left panel) and diaphragm thickening fraction (right panel) measured by ultrasound at the moment of switch to pressure-support ventilation.
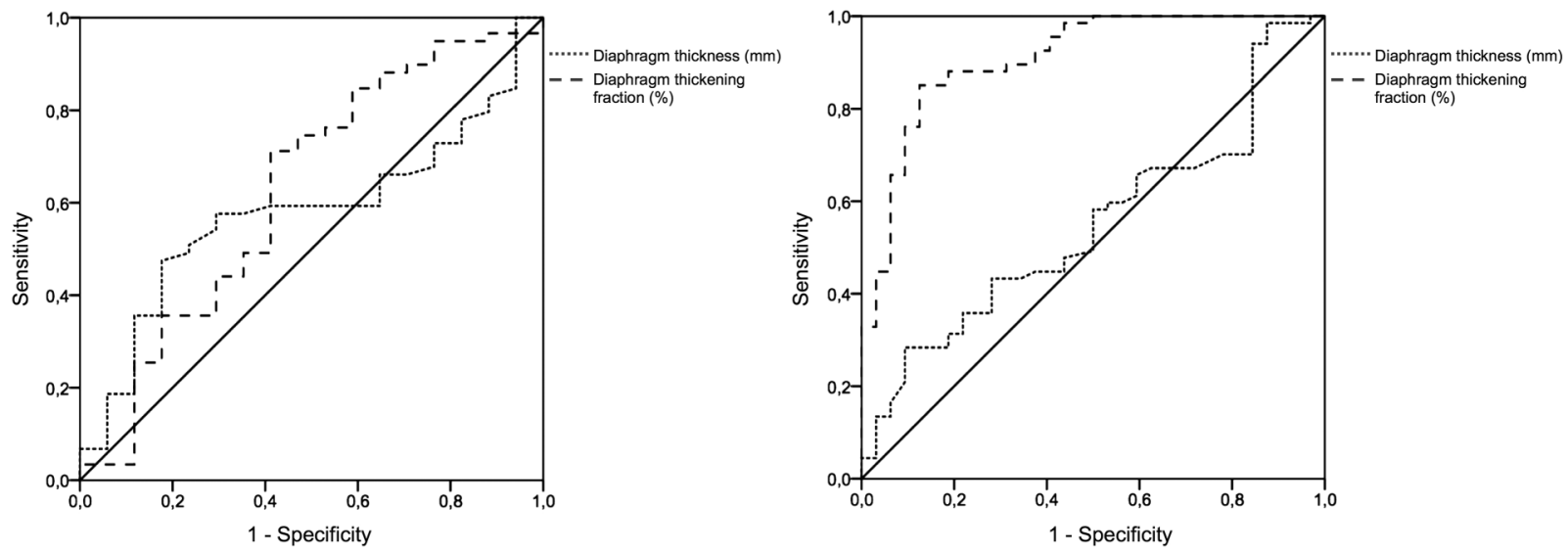

Figure 4 Receiver operating characteristic (ROC) curve for the diagnosis of diaphragm dysfunction (defined as a change in endotracheal tube pressure induced by bilateral anterior magnetic phrenic nerve stimulation $<11 \mathrm{~cm} \mathrm{H} \mathrm{H}_{2} \mathrm{O}$ ) for end-expiratory diaphragm thickness (dotted line) and diaphragm thickening fraction (dashed line), on initiation of mechanical ventilation (left panel) and switch to pressure support ventilation (right panel).

Table 3 Clinical outcomes according to the presence of diaphragm dysfunction at switch to pressure support ventilation

\begin{tabular}{|c|c|c|c|c|c|c|}
\hline & \multicolumn{3}{|c|}{$\begin{array}{l}\text { Diaphragm dysfunction defined as Ptr,stim }<11 \\
\mathrm{~cm} \mathrm{H}_{2} \mathrm{O}\end{array}$} & \multicolumn{3}{|c|}{ Diaphragm dysfunction defined as TFdi $<29 \%$} \\
\hline & Yes $(n=67)$ & No $(n=32)$ & p Value & Yes $(n=61)$ & No $(n=38)$ & p Value \\
\hline Length of ICU stay, days & $10(6-16)$ & $6(3-12)$ & 0.05 & $10(6-17)$ & $6(4-11)$ & 0.02 \\
\hline Length of MV, days & $7(5-11)$ & $4(1-7)$ & 0.04 & $7(5-12)$ & $5(2-8)$ & 0.02 \\
\hline ICU deaths, n (\%) & $15(22)$ & $2(6)$ & 0.05 & $16(26)$ & $1(3)$ & 0.002 \\
\hline Hospital deaths, n (\%) & $18(27)$ & $3(9)$ & 0.05 & $19(31)$ & $2(5)$ & 0.002 \\
\hline Remaining days of MV after measurement, days & $3(1-5)$ & $0(0-1)$ & 0.04 & $2(1-5)$ & $0(0-3)$ & 0.04 \\
\hline
\end{tabular}

predicted remaining duration of MV: Ptr,stim and TFdi. e-table 4 shows the factors associated with ICU mortality identified by univariate analysis. By multiple linear regression model analysis, three of these factors independently predicted ICU mortality: Ptr,stim, TFdi and $\mathrm{PaO}_{2} / \mathrm{FiO}_{2}$ ratio. e-table 5 shows the factors associated with hospital mortality identified by univariate analysis. By multiple linear regression model analysis, three of these factors independently predicted remaining duration of MV: Ptr, stim, TFdi and $\mathrm{PaO}_{2} / \mathrm{FiO}_{2}$ ratio.

\section{DISCUSSION}

Our main findings are as follows: in a cohort of mechanically ventilated patients (1) right diaphragm thickness was unrelated to diaphragm pressure generation capacity, (2) at the moment of switch to PSV, the thickening ratio of the right diaphragm was a reliable marker of diaphragm function and (3) diaphragm dysfunction at switch to PSV was associated with adverse clinical outcomes. This is the first study to systematically investigate the relationship between ultrasound-derived 
markers of diaphragm function and an established method of diaphragm contractility evaluation.

These results help to clarify the validity of ultrasound-derived measurements of diaphragm function in the ICU population, delineate the circumstances under which these measurements should be performed in order to truly represent diaphragm function and suggest that the prognostic value of diaphragm function in this population is possible to assess using ultrasound.

\section{Relationship between diaphragm thickness and Ptr,stim}

Our finding that diaphragm thickness was unrelated to its pressure generation ability can seem surprising, since a decrease in muscle thickness could intuitively be expected to translate into muscle weakness. When measured serially, diaphragm thickness has been shown to decrease with ongoing $\mathrm{MV}^{18}{ }^{28}$ presumably reflecting muscle atrophy and weakness. However, several findings cast doubt on the putative relationship between diaphragm thickness and its strength. In a study investigating the evolution of diaphragm thickness of critically ill patients during their first week of $\mathrm{MV}^{18}$ low diaphragm contractile activity (estimated using TFdi) was associated with both a decline and an increase in diaphragm thickness. It is possible that an increase in diaphragm thickness may therefore represent a pathological process such as tissue oedema, rather than an increase in functional muscle mass. A report on the distribution of diaphragm thickness in a large cohort of healthy subjects showed that a value of $0.17 \mathrm{~cm}$ represented the lower limit of normal of the distribution for the right hemidiaphragm. ${ }^{29}$ However, among patients with long-standing phrenic paresis or diaphragmatic myositis, a proportion of patients present with diaphragm thickness values above this threshold. ${ }^{30-33}$ In light of this, we believe that these findings, coupled with our own, cast serious doubt on the validity and usefulness of diaphragm thickness as a relevant marker of diaphragm function, at least in the ICU setting.

\section{Relationship between diaphragm TFdi and Ptr,stim}

In contrast, we found a strong relationship between diaphragm twitch pressure and TFdi, but only when measured at switch to PSV. We hypothesise that the reason we observed such a strong correlation at that moment but not at the initiation of MV was the presence of a much higher prevalence of spontaneous breathing effort in the former, but not in the latter. Indeed, it seems intuitive to suppose that without active breathing efforts, TFdi cannot be expected to act as a dynamic physiological estimator of diaphragm function. Interestingly, some degree of diaphragm thickening could still be observed in the majority of patients under ACV, although the mean value was much lower than in patients under PSV. This finding should alert clinicians to the fact that the observation of a non-zero diaphragm thickening in patients under MV should not systematically be considered a reliable estimator of the underlying Ptr,stim. In this regard, our findings echo those of others, in which even patients under neuromuscular blockade showed a small but measurable degree of diaphragm thickening. ${ }^{20}$ In these patients, and in patients under ACV, it is possible that the passive inflation of the lung and mechanical displacement of the diaphragm, as well as weak or partial respiratory efforts may be partly responsible for the observed thickening of the diaphragm, especially at higher lung volumes. In addition, it should be kept in mind that significant alterations in end-expiratory diaphragm thickness caused by atrophy or oedema may alter the value of TFdi, which could impair its relationship with Ptr,sim, although this phenomenon may be mitigated if end-expiratory and end-inspiratory thicknesses are similarly affected. Further studies on this issue are required.

A recent study which compared various markers of diaphragm activity in the prediction of weaning outcome also found a significant relationship between TFdi and diaphragm function measured using magnetic stimulation of the phrenic nerves, although it was lower than in our study. ${ }^{34}$

\section{Relationship between EXdi and Ptr,stim}

EXdi was not correlated to Ptr,stim at the initiation of MV, but only moderately correlated to Ptr,stim at switch to PSV. This finding may be related to the fact that EXdi is dependent on the degree of active diaphragm contraction, and on the passive displacement of the diaphragm induced by the ventilator.

\section{Relationship between diaphragm function and outcome}

The finding that diaphragm function is related to length of $\mathrm{MV}$, duration of stay and mortality of critically ill patients is not new, but had until now mainly been evaluated using phrenic nerve stimulation techniques, ${ }^{156}$ which cannot be routinely used in clinical practice. Our study is the first to report the significant relationship between TFdi and clinically relevant outcomes such as length of MV and ICU and hospital stay and mortality. Of note, our finding that a higher TFdi value is associated with a decreased remaining duration of MV echoes the findings of others, who showed that TFdi could act as an important predictor of weaning outcome. ${ }^{14} \quad 15 \quad 17 \quad 22$ Our results add the important finding that these clinically important outcomes can be accurately assessed using ultrasound, a simple, non-invasive and rapid technique that is widely available. It remains unclear whether diaphragm dysfunction itself has a causative effect on the prognosis of ICU patients, or if its presence should be seen as an organ dysfunction, implying a more severe underlying disease. Our results do not allow that question to be answered, but fuel the need for additional studies evaluating the putative role of diaphragmatic function in the evolution of ICU patients.

\section{Strength and limitations}

The strengths of our study include the inclusion of a large number of patients, allowing for a meaningful comparison of ultrasound variables with the reference method. In addition, the systematic use of bilateral magnetic stimulation of the phrenic nerves as a reference method lends strength to our results, as this technique allows an objective and non-volitional evaluation of diaphragm function that is more reliable than other methods such as the measurement of maximal inspiratory pressures, especially in the ICU setting. ${ }^{35}$ We measured TFdi during tidal breathing, which has the advantage of being non-volitional and possible to use even in sedated or non-cooperative patients. Our choice of performing measurements at the moment of switching to PSV was based our belief that this moment had clinical relevance, as it likely represents an individualised moment where patients begin to recover from their underlying illness. Our findings that diaphragm function measured at this time point had prognostic implications support the clinical usefulness of the evaluation of diaphragm function even after several days of MV.

We acknowledge several limitations to our study. First, the conditions in which we performed our measurements must be taken into account when generalising our results. When measured under MV, TFdi is related to neural respiratory drive, and can be influenced by the level of diaphragm unloading and therefore by the level of assistance provided by the ventilator. $^{36} 37$ We did not formally measure neural respiratory drive to the diaphragm or work of breathing in our study, and 
although it is possible that these variables may have influenced our measurement of TFdi, we believe this must not have been a significant confounding factor because of (1) the fact that most patients under ACV had no spontaneous breathing efforts and (2) the fact that patients under PSV received individualised pressure support levels targeted at a tidal volume that minimised situations of increased or decreased respiratory drive such as overassistance or underassistance. These subjects therefore probably displayed a relatively homogeneous degree of respiratory drive. We need to emphasise, however, that our results may not be generalisable to a population in which the prevailing ventilator settings or respiratory state result in abnormally increased or decreased work of breathing such as respiratory distress, weaning trials or in situations of overassistance and underassistance. Second, the use of a Ptr,stim cut-off value of $11 \mathrm{~cm} \mathrm{H}_{2} \mathrm{O}$ to define the presence of diaphragmatic dysfunction can be questioned, but this value has been reported as indicative of diaphragm weakness. ${ }^{1} 38$ Third, our study was performed in a medical ICU. As such, it is possible that our results would not be directly applicable to patients recovering from surgery, especially major thoracic or abdominal surgeries, which could alter the measurement of Ptr,stim and TFdi. Fourth, as we did not formally confirm the supramaximal nature of phrenic stimulation in our patients, we cannot rule out that Ptr,stim was underestimated in some of them, which could have influenced our results. In the present study, we deliberately opted to omit supramaximality testing to avoid inducing excessive discomfort. Although we acknowledge this issue as requiring caution, we note that the stimulation protocol that we used has been reported to produce supramaximal stimulations in many cases, including in ICU patients. ${ }^{4} 92425$ We also wish to point out that independently of whether the stimulations delivered were supramaximal or not, the Ptr,stim threshold that we used was significantly and independently associated with adverse clinical outcomes. From a pragmatic point of view and with regards to the transposability of our results, this means that performing phrenic stimulation at the maximal output of the device we used can provide clinically useful data. Fifth, from a statistical viewpoint, the comparison of two ROC curves should be made with caution when they are crossing. However, this is unlikely to be significant in our results as (1) both diaphragm thickness and TFdi had non-significant AUROC curves for the prediction of diaphragm dysfunction under ACV and (2) under PSV, where the comparison is most relevant, the curves do not cross. Finally, we limited our ultrasound evaluation to the right hemidiaphragm because of the higher interobserver reliability of observations on this side, which is probably related to the better acoustic window provided by the underlying liver tissue.

\section{CONCLUSION}

In a cohort of critically ill, mechanically ventilated patients, we found no significant relationship between diaphragm thickness and its contractile capacity, suggesting that diaphragm thickness should not be used as an estimator of diaphragm contractility in the ICU setting. In contrast, we found a strong relationship between TFdi and Ptr,stim, when measured in patients receiving a partial mode of ventilator assistance, suggesting that TFdi can be used to diagnose diaphragm dysfunction in this setting. This is all the more relevant since the presence of such a dysfunction at this stage of ICU stay was associated with a poorer prognosis. Taken together, these results provide evidence that TFdi can be used as a reliable estimator of diaphragm function in this setting. Beyond prognostic purposes, this could prove useful for the management of indications and the follow-up of putative therapeutic approaches such as inspiratory muscle training ${ }^{39}$ or diaphragm pacing. ${ }^{1140}$

Contributors B-PD, MD and AD designed the study. MD coordinated the study. $M D, B-P D, S D$ and $J M$ were responsible for patient screening, enrolment and follow-up. MD, B-PD, TS and AD analysed the data. B-PD, MD, TS and AD wrote the manuscript. All authors had full access to all of the study data, contributed to draft the manuscript or revised it critically for important intellectual content, approved the final version of the manuscript, and take responsibility for the integrity of the data and the accuracy of the data analysis.

Funding B-PD was supported by a postgraduate fellowship grant from the Fondation du Centre Hospitalier de I'Université de Montréal (CHUM). MD was supported by The French Intensive Care Society (SRLF bourse de mobilité 2015); The 2015 Short Term Fellowship programme of the European Respiratory Society; The 2015 Bernhard Dräger Award for advanced treatment of ARF of the European Society of Intensive Care Medicine; The Assistance Publique Hôpitaux de Paris; The Fondation pour la Recherche Médicale (FDM 20150734498) and by Mitacs Globalink Sorbonne Universités. The clinical research of 'Département R3S' is supported by the programme 'investissement d'avenir ANR-10-AlHU 06' of the French Government.

Competing interests B-PD has received honoraria from GlaxoSmithKline, Boehringer Ingelheim, Astra Zeneca and Roche. AD has signed research contracts with Covidien, Maquet and Philips; he has also received personal fees from Covidien and MSD. MD received personal fees from Pulsion Medical System and Astra Zeneca. Relevant to the present study, TS has received personal fees from Lungpacer and is a member of the board of a research association that has received, over the past 10 years, unrestricted research grants from Maquet, Hamilton, Covidien and Philips; he is the head of a research unit (UMRS 1158) that has signed research contracts with Air Liquide Medical Systems, France; he is listed as inventor or co-inventor on several patents, granted or pending, describing a brain-ventilator interface.

Ethics approval Comité de Protection des Personnes lle de France VI.

Provenance and peer review Not commissioned; externally peer reviewed.

\section{REFERENCES}

1 Demoule $A$, Jung $B$, Prodanovic $H$, et al. Diaphragm dysfunction on admission to the intensive care unit. Prevalence, risk factors, and prognostic impact-a prospective study. Am J Respir Crit Care Med 2013;188:213-19.

2 Hooijman PE, Beishuizen A, Witt CC, et al. Diaphragm muscle fiber weakness and ubiquitin-proteasome activation in critically ill patients. Am J Respir Crit Care Med 2015; 191:1126-38.

3 Laghi F, Cattapan SE, Jubran A, et al. Is weaning failure caused by low-frequency fatigue of the diaphragm? Am J Respir Crit Care Med 2003;167:120-7.

4 Watson AC, Hughes PD, Louise Harris M, et al. Measurement of twitch transdiaphragmatic, esophageal, and endotracheal tube pressure with bilateral anterolateral magnetic phrenic nerve stimulation in patients in the intensive care unit. Crit Care Med 2001;29:1325-31.

5 Hermans G, Agten A, Testelmans D, et al. Increased duration of mechanical ventilation is associated with decreased diaphragmatic force: a prospective observational study. Crit Care 2010;14:R127.

6 Jaber $S$, Petrof BJ, Jung B, et al. Rapidly progressive diaphragmatic weakness and injury during mechanical ventilation in humans. Am J Respir Crit Care Med 2011;183:364-71.

7 Maher J, Rutledge F, Remtulla $\mathrm{H}$, et al. Neuromuscular disorders associated with failure to wean from the ventilator. Intensive Care Med 1995;21:737-43.

8 Vassilakopoulos T, Zakynthinos S, Roussos C. The tension-time index and the frequency/tidal volume ratio are the major pathophysiologic determinants of weaning failure and success. Am J Respir Crit Care Med 1998;158:378-85.

9 Supinski GS, Callahan LA. Diaphragm weakness in mechanically ventilated critically ill patients. Crit Care 2013;17:R120.

10 Martin AD, Joseph AM, Beaver TM, et al. Effect of intermittent phrenic nerve stimulation during cardiothoracic surgery on mitochondrial respiration in the human diaphragm. Crit Care Med 2014;42:e152-6.

11 Masmoudi H, Coirault C, Demoule A, et al. Can phrenic stimulation protect the diaphragm from mechanical ventilation-induced damage? Eur Respir $J$ 2013;42:280-3.

12 Laghi F, Shaikh H. Preventing ventilator-induced diaphragmatic dysfunction with phrenic nerve stimulation. Crit Care Med 2014:42:492-4.

13 American Thoracic Society/European Respiratory Society. ATS/ERS Statement on Respiratory Muscle Testing. Am J Respir Crit Care Med 2002;166:518-624.

14 DiNino E, Gartman EJ, Sethi JM, et al. Diaphragm ultrasound as a predictor of successful extubation from mechanical ventilation. Thorax 2014:69:423-7.

15 Ferrari G, De Filippi G, Elia F, et al. Diaphragm ultrasound as a new index of discontinuation from mechanical ventilation. Crit Ultrasound J 2014;6:8. 
16 Jiang JR, Tsai TH, Jerng JS, et al. Ultrasonographic evaluation of liver/spleen movements and extubation outcome. Chest 2004;126:179-85.

17 Kim WY, Suh HJ, Hong SB, et al. Diaphragm dysfunction assessed by ultrasonography: influence on weaning from mechanical ventilation. Crit Care Med 2011;39:2627-30.

18 Goligher EC, Fan E, Herridge MS, et al. Evolution of Diaphragm Thickness During Mechanical Ventilation: Impact of Inspiratory Effort. Am J Respir Crit Care Med 2015;192:1080-8.

19 Levine S, Nguyen T, Taylor N, et al. Rapid disuse atrophy of diaphragm fibers in mechanically ventilated humans. N Engl J Med 2008;358:1327-35.

20 Goligher EC, Laghi F, Detsky ME, et al. Measuring diaphragm thickness with ultrasound in mechanically ventilated patients: feasibility, reproducibility and validity. Intensive Care Med 2015;41:642-9.

21 Dubé B-P, Dres $M$, Prodanovic $H$, et al. Reliability of diaphragm ultrasound to detect diaphragm dysfunction in critically ill patients. Eur Resp J 2015;46(Suppl 59): OA4957.

22 Dres M, Dubé BP, Mayaux J, et al. Coexistence and Impact of Limb Muscle and Diaphragm Weakness at Time of Liberation From Mechanical Ventilation in Medical ICU Patients. Am J Respir Crit Care Med 2017:195:57-66.

23 Cattapan SE, Laghi F, Tobin MJ. Can diaphragmatic contractility be assessed by airway twitch pressure in mechanically ventilated patients? Thorax 2003;58:58-62.

24 Mills $\mathrm{GH}$, Kyroussis D, Hamnegard $\mathrm{CH}$, et al. Bilateral magnetic stimulation of the phrenic nerves from an anterolateral approach. Am J Respir Crit Care Med 1996:154:1099-105.

25 Polkey MI, Kyroussis D, Hamnegard C-H, et al. Diaphragm strength in chronic obstructive pulmonary disease. Am J Respir Crit Care Med 1996;154:1310-7.

26 Matamis D, Soilemezi E, Tsagourias M, et al. Sonographic evaluation of the diaphragm in critically ill patients. Technique and clinical applications. Intensive Care Med 2013;39:801-10.

27 Levy MM, Fink MP, Marshall JC, et al. 2001 SCCM/ESICM/ACCP/ATS/SIS International Sepsis Definitions Conference. Intensive Care Med 2003;29:530-8.

28 Grosu HB, Lee $\mathrm{Yl}$, Lee J, et al. Diaphragm muscle thinning in patients who are mechanically ventilated. Chest 2012;142:1455-60.
29 Boon AJ, Harper CJ, Ghahfarokhi LS, et al. Two-dimensional ultrasound imaging of the diaphragm: quantitative values in normal subjects. Muscle Nerve 2013;47:884-9.

30 Summerhill EM, El-Sameed YA, Glidden TJ, et al. Monitoring recovery from diaphragm paralysis with ultrasound. Chest 2008;133:737-43.

31 Yoshioka Y, Ohwada A, Sekiya M, et al. Ultrasonographic evaluation of the diaphragm in patients with amyotrophic lateral sclerosis. Respirology 2007;12:304-7.

32 Gottesman E, McCool FD. Ultrasound evaluation of the paralyzed diaphragm. Am J Respir Crit Care Med 1997;155:1570-4.

33 Bruin PFD, Ueki J, Bush A, et al. Diaphragm thickness and inspiratory strength in patients with Duchenne muscular dystrophy. Thorax 1997:52:474-5.

34 Jung B, Moury PH, Mahul M, et al. Diaphragmatic dysfunction in patients with ICU-acquired weakness and its impact on extubation failure. Intensive Care Med 2016:42:853-61.

35 Supinski GS, Westgate P, Callahan LA. Correlation of maximal inspiratory pressure to transdiaphragmatic twitch pressure in intensive care unit patients. Critical Care 2016;20:1-15.

36 Vivier E, Mekontso Dessap A, Dimassi S, et al. Diaphragm ultrasonography to estimate the work of breathing during non-invasive ventilation. Intensive Care Med 2012;38:796-803.

37 Umbrello M, Formenti $\mathrm{P}$, Longhi $\mathrm{D}$, et al. Diaphragm ultrasound as indicator of respiratory effort in critically ill patients undergoing assisted mechanical ventilation: a pilot clinical study. Crit Care 2015;19:161.

38 Mills GH, Ponte J, Hamnegard $\mathrm{CH}$, et al. Tracheal tube pressure change during magnetic stimulation of the phrenic nerves as an indicator of diaphragm strength on the intensive care unit. Br J Anaesth 2001;87:876-84.

39 Elkins M, Dentice R. Inspiratory muscle training facilitates weaning from mechanica ventilation among patients in the intensive care unit: a systematic review. J Physiother 2015;61:125-34

40 Reynolds SC, Meyyappan R, Thakkar V, et al. Mitigation of Ventilator-Induced Diaphragm Atrophy by Transvenous Phrenic Nerve Stimulation. Am J Respir Crit Care Med 2017:195:339-48. 